\title{
Rotation intervals of endomorphisms of the circle
}

\author{
R. BAMON, I. P. MALTA, M. J. PACIFICO AND F. TAKENS
}

Departamento de Matemática, Universidad de Chile, Casilla 653-Santiago, Chile; Departamento de Matemática, Pontifícia Universidade Católica do Rio de Janeiro, Rua Marquês de São Vicente, 225, Casa B, CEP 22.453-Gávea-Rio de Janeiro RJ_Brazil; Instituto de Matemática, Universidade Federal do Rio de Janeiro, Caixa Postal 68530, CEP 21.910-Rio de Janeiro RJ-Brazil; Mathematisch Instituut, Postbus 800, 9700 AV Groningen, The Netherlands

(Received 16 November 1983)

Abstract. The rotation number of a diffeomorphism $f: S^{1} \rightarrow S^{1}$, with lift $F: \mathbb{R} \rightarrow \mathbb{R}$ is defined as $\lim _{n \rightarrow \infty}\left(F^{n}(x)-x\right) / n$. We investigate the case where $f$ is an endomorphism. Then this limit may not exist and may depend on $x$. We investigate the set of limit points of $\left(F^{n}(x)-x\right) / n, n \rightarrow \infty$, as a function of $x$.

\section{Introduction and statement of results}

Let $\operatorname{End}_{1}^{0}\left(S^{1}\right)$ be the set of continuous endomorphisms of degree one of the circle. Given $f \in \operatorname{End}_{1}^{0}\left(S^{1}\right)$, let $F$ be a lift of $f$ to $\mathbb{R}$, that is, $f \circ \pi=\pi \circ F$, where $\pi: \mathbb{R} \rightarrow S^{1}$ is the natural projection. Since $f$ is of degree one, we have

$$
F(x+1)=F(x)+1 \quad \text { for all } x \in \mathbb{R} \text {. }
$$

If $f$ is a homeomorphism it is well known that the limit of $\left(F^{n}(x)-x\right) / n$ for $n \rightarrow \infty$, exists, does not depend on $x$ and defines the rotation number of $f$. In the general case of endomorphisms this limit may not exist. In [2] Newhouse, Palis and Takens introduced the concept of rotation set of endomorphisms. It is defined by

$$
\rho(f)=\text { closure }\left\{\rho^{+}(f, z), z \in S^{1}\right\}
$$

where

$$
\rho^{+}(f, z)=\lim _{n} \sup \frac{F^{n}(x)-x}{n}
$$

$\pi(x)=z$. They also proved that $\rho(f)$ is an interval. Clearly $\rho(f)$ is defined up to translations by integers. In [1] R. Ito proved that each $\alpha \in \rho(f)$ is realized as the rotation number of some point in $S^{1}$, in the sense that for some $z \in S^{1}$, $\lim _{n \rightarrow \infty}\left(F^{n}(x)-x\right) / n=\alpha, \pi(x)=z$.

Here we define the rotation set $\rho(f, z)$ of $z \in S^{1}$ as the set of limit points of the sequence $\left(F^{n}(x)-x\right) / n$, where $\pi(x)=z$. Observe that $\rho(f, z) \subseteq \rho(f)$. 
The purpose of this paper is to give a complete description of all rotation sets $\rho(f, z)$ in terms of the rotation interval $\rho(f)$.

THEOREM. If $f \in \operatorname{End}_{1}^{0}\left(S^{\mathbf{l}}\right)$ then:

(i) $\rho(f, z)$ is a closed subinterval of $\rho(f)$ for all $z \in S^{1}$;

(ii) given $[\alpha, \beta] \subseteq \rho(f), \alpha \leq \beta$, there exists $z \in S^{\prime}$ such that $\rho(f, z)=[\alpha, \beta]$.

In $\S 2$ we introduce the notion of positive local unstable manifold for periodic points of endomorphisms as well as the concept of fundamental domains which play a key rôle in the proof of the theorem. In $\S 3$ we prove some technical lemmas and finally, the proof of the theorem is given in $\$ 4$.

We are grateful to IMPA for its very kind hospitality.

\section{Preliminaries}

Let $f \in \operatorname{End}_{1}^{0}\left(S^{1}\right)$. If $z_{0}$ is a fixed point of $f$, let $F$ be a lift of $f$ with $F(x)=x$ if $\pi(x)=z_{0}$. Consider the set

$$
A^{+}=\left\{z \in S^{1} ; F(x)>x, \pi(x)=z\right\} .
$$

If there exists a component $U \subset A^{+}, \bar{U}=\left[z_{0}, z\right]$, we define the local positive unstable manifold of $z_{0}, W_{\text {loc }}^{u+}\left(z_{0}\right)$ as the set $\left\{z_{0}\right\} \cup U$. Otherwise we define $W_{\text {loc }}^{u+}\left(z_{0}\right)=\left\{z_{0}\right\}$. Note that even for a fixed point $z_{0}$ where $f$ is expanding but orientation reversing, $W_{\text {loc }}^{u+}\left(z_{0}\right)=\left\{z_{0}\right\}$. The (positive) unstable manifold of $z_{0}, W^{u+}\left(z_{0}\right)$, is defined as

$$
W^{u+}\left(z_{0}\right)=\bigcup_{j \geq 0} f^{i}\left(W_{\text {loc }}^{u+}\left(z_{0}\right)\right)
$$

If $\pi\left(x_{0}\right)=z_{0}$ we define $W_{\text {loc }}^{u+}\left(x_{0}\right)$ as the lift of $W_{\text {loc }}^{u+}\left(z_{0}\right)$ which contains $x_{0}$.

If $z_{0}$ is a periodic point of $f$ with period $q, W_{\mathrm{loc}}^{u+}\left(z_{0}\right)$ and $W^{u+}\left(z_{0}\right)$ are defined as above considering $z_{0}$ as a fixed point of $f^{q}$.

In order to define a fundamental domain in $W_{\text {loc }}^{u+}\left(z_{0}\right)$, we proceed as follows. Take a sequence $\left\{d_{i}\right\}_{i \in \mathbb{N}} \subset W_{\text {loc }}^{u+}\left(z_{0}\right)$ such that $f\left(d_{i+1}\right)=d_{i}$; so $d_{i}$ converges to $z_{0}$. We define $D_{i}=$ interval $\left[d_{i}, d_{i-1}\right]$. We call such an interval $D_{i}$, so that $f$ maps the interval $\left[z_{0}, d_{i}\right]$ inside $W_{\text {loc }}^{u+}\left(z_{0}\right)$, a fundamental domain in $W_{\text {loc }}^{u+}\left(z_{0}\right)$. Observe that since $f$ is an endomorphism, there may exist $s \in D_{i+1}$ such that $f(s) \notin D_{i}$. So the notion of fundamental domain here is weaker than the usual one for diffeomorphisms. In particular, even if $W^{u+}\left(z_{0}\right)=S^{1}$ and if $D \subset W^{u+}(z)$ is a fundamental domain it may not follow that for some $j, f^{j}(D)=S^{1}$. However we shall prove:

Proposition 1. Let $f \in \operatorname{End}_{1}^{0}\left(S^{1}\right)$ and $p / q \in$ int $\rho(f),(p, q)=1$. Then there exists a periodic point $z$ with rotation number $p / q$ and period $q$, and a fundamental domain $D \subset W^{u+}(z)$ such that $f^{j}(D)=S^{\prime}$ for some $j \in \mathbb{N}$.

Proof. It is enough to prove the case when $p=0$ and $q=1$. Let $C$ be the set of fixed points $z$ with rotation number zero, that is, $F(x)=x$ if $\pi(x)=z$ for $z \in C$. We claim that there exists $z=\pi(x) \in C$ such that

$$
F^{i_{0}}\left(W_{\text {loc }}^{u+}(x)\right) \supset[x, x+2] \quad \text { for some } i_{0} \geq 1 .
$$

Suppose that this is not true. We will prove that this assumption implies $\rho^{+}(f, w) \leq 0$ for all $w \in S^{1}$, which is a contradiction. In fact if $y \in W_{\text {loc }}^{u+}(x), \pi(x) \in C$ then $y \geq x$ 
and so $F^{n}(y)<y+2$ for all $n \geq 1$. Thus $\rho^{+}(f, \pi(y)) \leq 0$ for $\pi(y) \in W_{\text {loc }}^{u+}(z), z \in C$. Since $\pi(y) \in W_{\text {loc }}^{u+}(z), z \in C$, if and only if $F(y) \geq y$, it remains to consider $y$ such that $F(y)<y$. In this case we have $F^{n}(y)<y$ for all $n \geq 1$ or there exists $n_{0}$ such that $f^{n_{0}}(\pi(y)) \in W_{\text {loc }}^{u+}(z)$ for some $z \in C$. So we also have $\rho^{+}(f, \pi(y)) \leq 0$.

Let $z_{0}=\pi\left(x_{0}\right) \in C$ and $i_{0} \geq 1$ be as in the claim. Take $y_{0} \in W_{\text {loc }}^{u+}\left(x_{0}\right)$ such that $F^{i_{0}}\left(y_{0}\right)=x_{0}+2$. Let

$$
y_{1}=\sup \left\{y \in\left[x_{0}, y_{0}\right] \text { such that } F^{i_{0}}(y)=x_{0}+1\right\} .
$$

Then $F^{i_{0}}(y)>x_{0}+1$ for $y_{1}<y \leq y_{0}$. Thus if $W$ is any neighbourhood of $w=\pi\left(y_{1}\right)$ then $f^{i}(W)$ covers a neighbourhood of $z_{0}$ in $W_{\text {loc }}^{u+}\left(z_{0}\right)$. It is possible to choose $\left\{d_{i}\right\}_{i \in \mathbb{N}} \subset W_{\text {loc }}^{u+}\left(z_{0}\right)$ with $f\left(d_{i+1}\right)=d_{i}$ and $w \in\left[d_{2}, d_{1}\right]$. Clearly, for $i$ big enough, $D=$ $\left[d_{i+1}, d_{i}\right]$ is a fundamental domain with the required property.

\section{Itineraries and technical lemmas}

Let $f \in \operatorname{End}_{1}^{0}\left(S^{1}\right)$ with $\rho(f)=[a, b], a<b$, and $\left\{p_{i} / q_{i}\right\}_{i \in \mathbb{N}} \subseteq$ int $(\rho(f))$ be a sequence of rational numbers. For each $i \in \mathbb{N}$, let $z_{i} \in S^{1}$ be a periodic point with rotation number $p_{i} / q_{i}$ and period $q_{i}$ such that $W^{u+}\left(z_{i}\right)=S^{1}$. Let $D_{i}$ be a fundamental domain for $W^{u+}\left(z_{i}\right)$ and $j_{i} \in \mathbb{N}$ such that $f^{j_{i}}\left(D_{i}\right)=S^{1}$. Given any sequence $(n)_{\nu}=\left(n_{1}, n_{2}, \ldots\right)$ of positive integers, where $\nu$ is the length of the sequence if it is finite or $\nu=\infty$ if it is infinite, such that $n_{i}=r_{i} q_{i}$ with $r_{i} \in \mathbb{N}$, we define for $0 \leq i-1<\nu$

$$
N_{i}=n_{1}+j_{1}+\cdots+n_{i-1}+j_{i-1}+n_{i} \text { and } J_{i}=N_{i}+j_{i}
$$

Put $J_{0}=0$. We say that $z=\pi(y) \in W_{\mathrm{loc}}^{u+}\left(z_{1}\right)$ has itinerary $(n)_{\nu}$ with respect to $\left(z_{i}, j_{i}\right)$ if for each $0 \leq i<\nu$ there exists $x_{i+1} \in \pi^{-1}\left(z_{i+1}\right)$ such that

$$
F^{J_{i}+k q_{i+1}}(y)-k p_{i+1} \in W_{\mathrm{loc}}^{u+}\left(x_{i+1}\right) \quad \text { for } 0 \leq k q_{i+1} \leq n_{i+1},
$$

and

$$
f^{N_{i+1}}(z) \in D_{i+1}
$$

Clearly this definition does not depend on the lift $y$ of $z$. Observe that if $z$ satisfies the itinerary $(n)_{\nu}$ then the orbit of $z$ is successively $n_{i}$ iterates near the orbit of $z_{i}$. LEMMA 2. Given any sequence $(n)_{\nu}$ as above there exists a point $z \in W_{\mathrm{loc}}^{u+}\left(z_{1}\right)$ with itinerary $(n)_{\nu}$.

Proof. For each $0 \leq i-1<\nu$, let $A_{i}$ be defined by

$$
\begin{array}{r}
A_{i}=\left\{z \in W_{\mathrm{loc}}^{u+}\left(z_{i}\right): f^{n_{i}}(z) \in D_{i} \text { and there exist } y \in \pi^{-1}(z), x_{i} \in \pi^{-1}\left(z_{i}\right)\right. \\
\text { such that } \left.F^{k q_{i}}(y)-k p_{i} \in W_{\mathrm{loc}}^{u+}\left(x_{i}\right) \text { for } 0 \leq k q_{i} \leq n_{i}\right\} .
\end{array}
$$

Since $f^{n_{i}}\left(A_{i}\right)=D_{i}, A_{i}$ is a closed non-empty set. Let $L_{i}=f^{-j_{i}}\left(A_{i+1}\right) \cap D_{i}$. Since $f^{j_{i}}\left(D_{i}\right)=S^{1}$, we have that $L_{i}$ is a compact non-empty set. Now define $K_{1}=A_{1}$ and

$$
K_{i}=\left\{z \in A_{1} ; f^{N_{m}}(z) \in L_{m} \text { for } 1 \leq m<i\right\} \quad \text { for } i>1 .
$$

It follows immediately that each $K_{i}$ is a compact non-empty set and satisfies $K_{i+1} \subset K_{i}$. It is also clear that if $z \in K_{i}$ then $z$ has itinerary $(n)_{i}=\left(n_{1}, \ldots, n_{i}\right)$. Thus each $z \in \bigcap_{i=1}^{\infty} K_{i}$ has the prescribed itinerary. 
LeMMA 3. Given $q \in N$ and $\varepsilon>0$ there exists $n_{0} \geq 1$ such that for all $y \in \mathbb{R}$ and all $n \geq n_{0}$

$$
\left|\frac{F^{n+k}(y)-y}{n+k}-\frac{F^{n}(y)-y}{n}\right|<\varepsilon \quad \text { for } 0 \leq k \leq q .
$$

Proof. Let $R, S>0$ be such that $\left|F^{k}(y)-y\right|<R$ for all $y \in \mathbb{R}$ and $0 \leq k \leq q$ and $\left|\left(F^{n}(y)-y\right) / n\right|<S$ for all $y \in \mathbb{R}$ and $n \in \mathbb{N}$. Then

$$
\begin{aligned}
\left|\frac{F^{n+k}(y)-y}{n+k}-\frac{F^{n}(y)-y}{n}\right| & =\frac{1}{n(n+k)}\left|n\left(F^{k}\left(F^{n}(y)\right)-F^{n}(y)\right)-k\left(F^{n}(y)-y\right)\right| \\
& \leq \frac{R}{n+k}+\frac{k S}{n(n+k)} .
\end{aligned}
$$

So it is enough to take $n_{0} \in \mathbb{N}$ such that $R /(n+k)+k S / n(n+k)<\varepsilon$ for $n \geq n_{0}$ and $0 \leq k \leq q$.

LEMMA 4. Let $z \in S^{1}$ be a periodic point of $f$ with rotation number $p / q$ and $m \in \mathbb{N}$. Then given $\varepsilon>0$ there exists $n_{0} \geq 1$ such that for all $y \in \mathbb{R}$ and $x \in \pi^{-1}(z)$, if $n \geq n_{0}$ and

$$
\left|\left(F^{n+m}(y)-F^{m}(y)\right)-\left(F^{n}(x)-x\right)\right| \leq 2
$$

then

$$
\left|\frac{F^{n+m}(y)-y}{n+m}-p / q\right|<\varepsilon .
$$

Proof. Let $R>0$ be such that $\left|F^{m}(y)-y\right|<R$ for all $y \in \mathbb{R}$. Take $n_{0} \geq 1$ such that $(2+R) / n_{0}<\varepsilon / 2$ and $\left|\left(F^{n}(x)-x\right) /(n+m)-p / q\right|<\varepsilon / 2$ for $n \geq n_{0}$. Note that $n_{0}$ does not depend on the lift $x$ of $z$. Then, if $n \geq n_{0}$ we have

$$
\begin{aligned}
\left|\frac{F^{n+m}(y)-y}{n+m}-p / q\right| \leq & \left|\frac{\left(F^{n+m}(y)-F^{m}(y)\right)-\left(F^{n}(x)-x\right)}{n+m}\right| \\
& +\left|\frac{F^{m}(y)-y}{n+m}\right|+\left|\frac{F^{n}(x)-x}{n+m}-p / q\right| \\
& \leq \frac{2+R}{n+m}+\varepsilon / 2<\varepsilon .
\end{aligned}
$$

\section{Proof of the theorem}

To prove (i), since

$$
\frac{F^{n}(x)-x}{n}=\frac{\sum_{i=1}^{n} F^{i}(x)-F^{i-1}(x)}{n}
$$

and $\left\{F^{i}(x)-F^{i-1}(x)=F\left(F^{i-1}(x)\right)-F^{i-1}(x)\right\}_{i \in N}$ is uniformly bounded, it is enough to prove the following lemma:

LEMMA. Let $\left\{a_{i}\right\}_{i \in N}$ be a bounded sequence. Then the set of limit points of $(1 / n) \sum_{i=1}^{n} a_{i}$, as $n \rightarrow \infty$, is a closed interval.

Proof. Let $a_{+}, a_{-}$be the lim sup and the $\lim$ inf of $(1 / n) \sum_{i=1}^{n} a_{i}$, as $n \rightarrow \infty$. The set of limit points is clearly contained in $\left[a_{-}, a_{+}\right]$and contains $a_{-}$and $a_{+}$. We choose 
a subsequence $n_{j}$ such that

$$
\lim _{j \rightarrow \infty} \frac{1}{n_{2 j}} \sum_{i=1}^{n_{2 j}} a_{i}=a_{-}, \quad \lim _{j \rightarrow \infty} \frac{1}{n_{2 j+1}} \sum_{i=1}^{n_{2 j+1}} a_{i}=a_{+} .
$$

For an arbitrary point $a \in\left[a_{-}, a_{+}\right]$we choose a subsequence $n_{j}^{a}$ so that $n_{j} \leq n_{j}^{a} \leq n_{j+1}$ and so that, with this restriction, $\left(1 / n_{j}^{a}\right) \sum_{i=1}^{n_{j}^{a}} a_{i}$ is as near as possible to $a$. The fact that $\left(1 / n_{j}^{a}\right) \sum_{i=1}^{n_{j}^{a}} a_{i}$ converges to $a$ follows from the observation that

$$
\frac{1}{n} \sum_{i=1}^{n} a_{i}-\frac{1}{n+1} \cdot \sum_{i=1}^{n+1} a_{i}=\frac{1}{n(n+1)} \sum_{i=1}^{n} a_{i}-\frac{1}{n+1} a_{n+1}
$$

goes to zero for $n \rightarrow \infty$.

Proof of (ii). We may assume that $\rho(f)$ is an interval of positive length. Otherwise (ii) is trivial.

Let $[\alpha, \beta] \subseteq \rho(f)$ be a subinterval. Choose $\left\{\rho_{i}=p_{i} / q_{i}\right\}_{i \in \mathbb{N}}$ so that $\alpha<\rho_{i}<\beta$, $\lim _{i \rightarrow \infty} \rho_{2 i-1}=\alpha$ and $\lim _{i \rightarrow \infty} \rho_{2 i}=\beta$.

Let $\left\{\varepsilon_{i}\right\}_{i \in N}$ be a sequence of positive real numbers such that $\alpha<\rho_{i}-\varepsilon_{i}$ and $\rho_{i}+\varepsilon_{i}<\beta, i \geq 1$.

For each $i \geq 1$ let $z_{i} \in S^{1}, D_{i} \subset S^{1}$ and $j_{i} \in \mathbb{N}$ be given by proposition 1 ; that is:

(1) $z_{i}$ is a periodic point of $f$ with rotation number $\rho_{i}$ and period $q_{i}$.

(2) $D_{i}$ is a fundamental domain for $W^{u+}\left(z_{i}\right)$ such that $f^{j_{i}}\left(D_{i}\right)=S^{1}$.

(3) for each $i \geq 1$ there exists $k_{i} \geq 1$ such that

$$
\left|\frac{F^{k}(y)-y}{k}-\rho_{i}\right|<\varepsilon_{i}
$$

if $k \geq k_{i}, y$ and $F^{k^{\prime} q_{i}}(y)-k^{\prime} p_{i}$ belong to $W_{\text {loc }}^{u+}\left(x_{i}\right)$ for some $x_{i} \in \pi^{-1}\left(z_{i}\right)$, where $k=k^{\prime} q_{i}+r$ with $0 \leq r<q_{i}$.

This result is obtained using lemma 4 with $m=0, n=k^{\prime} q_{i}$, and lemma 3 with $q=q_{i}$.

Now we will construct, by induction, an itinerary $(n)_{\infty}$ such that a point $z \in S^{1}$ with this itinerary with respect to $\left(z_{i}, j_{i}\right)$ will satisfy $\rho(f, z)=[\alpha, \beta]$. We claim that there exists a sequence $(n)_{\infty}=\left(n_{1}, n_{2}, \ldots\right)$ such that if $z=\pi(y)$ has itinerary $(n)_{\infty}$ with respect to $\left(z_{i}, j_{i}\right)$, then for $i \geq 1$

$$
\left|\frac{F^{N_{i}+k}(y)-y}{N_{i}+k}-\rho_{i}\right|<\varepsilon_{i} \quad \text { for } 0 \leq k \leq k_{i}+j_{i-1} \text {, where } j_{0}=0 \text {. }
$$

From lemma 4 with $m=0$ and lemma 3 with $q=k_{1}$ we can obtain an integer $r_{1}$ such that $n_{1}=r_{1} q_{1}$ and condition (a) is satisfied with $i=1$ for all $y \in \mathbb{R}$ such that $z=\pi(y)$ has itinerary $(n)_{1}=\left(n_{1}\right)$. Suppose we have $(n)_{i}=\left(n_{1}, n_{2}, \ldots, n_{i}\right), i \geq 1$, such that all $z=\pi(y)$ with itinerary $(n)_{i}$ satisfy condition (a) for $1 \leq l \leq i$. As before, we can obtain, from lemma 4 with $m=J_{i}$ and lemma 3 with $q=k_{i+1}+j_{i}$, an integer $r_{i+1} \geq 1$ such that $n_{i+1}=r_{i+1} q_{i+1}$ and if

$$
\left|\left(F^{n_{i+1}+J_{i}}(y)-F^{J_{i}}(y)\right)-\left(F^{n_{i+1}}\left(x_{i+1}\right)-x_{i+1}\right)\right| \leq 2
$$

for some $x_{i+1} \in \pi^{-1}\left(z_{i+1}\right)$ then

$$
\left|\frac{F^{n_{i+1}+J_{i}+k}(y)-y}{n_{i+1}+J_{i}+k}-\rho_{i+1}\right|<\varepsilon_{i+1} \quad \text { for } 0 \leq k \leq k_{i+1}+j_{i} \text {. }
$$


Since $N_{i+1}=n_{i+1}+J_{i}$ it is clear that if $z=\pi(y)$ has itinerary $(n)_{i+1}=\left(n_{1}, \ldots, n_{i+1}\right)$ then $y$ satisfies (a) for $1 \leq l \leq i+1$. This proves the claim.

Thus if $z=\pi(y)$ has itinerary $(n)_{\infty}$ with respect to $\left(z_{i}, j_{i}\right)$ then $y$ satisfies condition (a) for all $i \geq 1$. We also have

$$
\lim _{k \rightarrow \infty} \frac{F^{N_{2 k-1}}(y)-y}{N_{2 k-1}}=\alpha \text { and } \lim _{k \rightarrow \infty} \frac{F^{n_{2 k}}(y)-y}{N_{2 k}}=\beta .
$$

We now prove that $\rho(f, z)=[\alpha, \beta]$. For this it is enough to prove that $\alpha \leq$ $\left(F^{n}(y)-y\right) / n \leq \beta$ for all $n \geq n_{1}$. Let $n>n_{1}$. Since $N_{i} \rightarrow \infty$ as $i \rightarrow \infty$ and $N_{i+1}>N_{i}$ there exist $i \geq 1$ and $0 \leq k<n_{i}+j_{i-1}$ such that $n=N_{i+1}+k$. The result is clear if $k \leq k_{i}+j_{i-1}$. If $k_{i}+j_{i-1}<k<n_{i}+j_{i-1}$ we have

$$
\begin{aligned}
\frac{F^{N_{i-1}+k}(y)-y}{N_{i-1}+k}= & \left(\frac{F^{N_{i-1}+j_{i-1}}(y)-y}{N_{i-1}+j_{i-1}}\right) \cdot \frac{N_{i-1}+j_{i-1}}{N_{i-1}+k} \\
& +\left(\frac{F^{k-j_{i-1}}\left(F^{N_{i-1}+j_{i-1}}(y)\right)-F^{N_{i-1}+j_{i-1}}(y)}{k-j_{i-1}}\right) \cdot \frac{k-j_{i-1}}{N_{i-1}+k}
\end{aligned}
$$

which is a convex combination of two numbers. Clearly the first one is in an $\varepsilon_{i-1}$-neighbourhood of $\rho_{i-1}$ and so it is in $[\alpha, \beta]$. Since $n_{i}>k-j_{i-1}>k_{i}$ we can apply condition (3) to conclude that the second one is in an $\varepsilon_{i}$-neighbourhood of $\rho_{i}$ and so it also belongs to $[\alpha, \beta]$. This completes the proof.

\section{REFERENCES}

[1] R. Ito. Rotation sets are closed. Math. Proc. Camb. Phil. Soc. 89 (1981), 107-111.

[2] S. Newhouse, J. Palis \& F. Takens. Bifurcations and stability of families of diffeomorphisms. IHES Publ. Math. 57 (1983), 5-71. 